\title{
Diversity of Hover flies (Insecta: Diptera: Syrphidae) with 3 New Record From High Hill Zone of Himachal Pradesh, India
}

\author{
Jayita Sengupta $^{1 *}$, Atanu Naskar ${ }^{1}$, Sumit Homechaudhuri ${ }^{3}$, Dhriti Banerjee ${ }^{4}$ \\ ${ }^{1}$ Senior Zoological Assistant, Diptera Section, Zoological Survey of India, Kolkata, India \\ ${ }^{2}$ Assistant Zoologist, Diptera Section, Zoological Survey of India, Kolkata, India \\ ${ }^{3}$ Professor, Department of Zoology, University of Calcutta, Kolkata, India \\ ${ }^{4}$ Scientist-E, Diptera Section, Zoological Survey of India, Kolkata, India \\ *Correspondence E-mail : jayitasengupta9@gmail.com*; atanudiptera@gmail.com; \\ sumithomechaudhuri@gmail.com; dhritibanerjee@gmail.com.
}

\begin{abstract}
The present study of High hill zone, Himachal Pradesh is an inimitable example of establishment of alternative pollinators as a main pollination service renderer. Present study is imperious at this juncture to explore the present status of syrphid fauna prevalent in this important high hill zone ecosystem, not only to provide a baseline data for future entomo faunal studies but also to discuss the potential role of hoverflies as pollinator in this ecosystem. Altogether, 19 species under 13 genera over 2 subfamilies have been reported from High hill zone of Himachal Pradesh. 3 species namely Syrphus (Syrphus) vitripennis Megen,1822, Eristalis (Eristalis) tenax (Linnaeus, 1758) and Eristalinus (Eristalinus) arvorum (Fabricius, 1787) are reported for the first time from this High hill zone as well as from the state of Himachal Pradesh. Their taxonomic keys and detail diagnosis of the reported species has been discussed along with the distributional pattern of these species along the High hill zone of Himachal Pradesh.
\end{abstract}

Keywords: Hover flies, New Record, High hill zone, Syrphidae, Taxonomy.

\section{Introduction}

Flies always have played important roles as primary and secondary consumers, nutrient recyclers, provide food for wildlife, and indicators of ecosystem utility (Anderson and Vondracek, 1999; Batzer et al., 1992; De Szalay and Resh, 1997; Euliss et al., 1991). The flies are directly pertinent to human society as Pollinator (Sengupta et al;2019), Vector (Maity et al;2019) or sometime as biocontrollers (Naskar et al.,2019) and nutrient recyclers in agrarian and wild ecosystems (Ghahari et al;2008). Undoubtedly, out of this diversification in role, one of the most appreciated one is the part of pollination (Sengupta et al;2018). Whenever dipteran pollination is being discussed, the role of charismatic Hover flies (Insecta: Diptera: Syrphidae) as an efficient pollinator is always highlighted. It is a group of pollinating insects which is one of the most speciose groups comprising of morphologically and biologically diverse organisms (Sengupta et al;2016b), makes up $4.90 \%$ of the known Dipteran fauna from India (Sengupta et al;2016a, Banerjee et al;2018). They are omnipresent and cosmopolitan in their distribution (Evenhuis \& 
Pape, 2019). As revealed from molecular and morphological evidences, the Hoverflies are monophyletic in evolutionary pattern (Teskey et al; 1981).

Role of hoverflies as natural paste controller (Potts et al;2006) is also widely accepted apart from their pollination services. Unfortunately, the contribution of this hovering flies rendered towards food security of surrounding ecosystem is highly neglected. (Ssymank et al;2008) Especially in high elevational landscape, where the first liner pollinator superheroes are not efficient enough (Sengupta et al; 2019). The elevational hazards, environmental harshness, reduced flight capability, loss of partial pressure of oxygen etc together act as a barrier for the main pollinators of agro ecosystems including honey bees, Lepidoptera, ladybird beetles etc. (Mani,2013). Here lies the importance of hoverflies as an alternative pollinator in all of this agricultural landscape of higher altitudinal gradient. From agro economic aspect in the present research, the study area of Himachal Pradesh has been also classified in four zones namely Shivalik Hill zone (601-1200 mt), Mid Hill zone (1201-1800 mt), High Hill zone (1801-2200 mt), Cold \& Dry Zone (2201-6500 $\mathrm{mt})$ (Joshi et al;2013). Present research has been based upon the data collected from throughout survey from High hill zone of Himachal Pradesh in the year 2015-2018 in the elevational range of 1801 to $2,200 \mathrm{mt}$. The present study of High hill zone, Himachal Pradesh is an inimitable example of establishment of alternative pollinators as a main pollination service renderer. Present study is imperious at this juncture to explore the present status of syrphid fauna prevalent in this important high hill zone ecosystem, not only to provide a baseline data for future entomo faunal studies but also to discuss the potential role of hoverflies as pollinator in this ecosystem.

\section{Materials and Methods}

Syrphid fauna was collected from the field during day time by using insect sweep nets as well as specimens were also collected from different traps including. The collected samples are narcotized by using ethyl acetate and stored for further study in insect envelopes in the field. After bringing the specimens back in laboratory, specimens are pinned and kept in insect boxes. Identification of the adults followed the keys of Miranda et al; 2013, Vockeroth 1992, Thompson et al; 1982, Teskey et al; 1981; and Brunetti 1907 keeping in mind the recent nomenclatural changes (Evenhuis \& Pape, 2019). All the identified specimens were deposited in the designated repository of National Zoological Collection, Diptera section, Zoological Survey of India, Kolkata. The graphical representations here were made by using Microsoft Excel 2016. The 3D map of the study area has been generated by using ARC GIS software version 10.1. The photograph of habitus and insect body and parts were taken by using Leica Microscope M205A, where 0.32x Acro lense was used for for habitus photography and PLANAPO 1.0X lense was used for the photography of body parts and Nikon D7000 (Nikkor normal and macro lens) were used for field photographs.

\section{Results}

The current study includes the total list of available syrphid species from High hill zone of Himachal Pradesh along with their systematic account, diagnostic taxonomic key, and distributional affinity in India as well as in all other zoogeographical realms. This study altogether recorded 19 species of hoverflies under 13 genera over 2 subfamilies. Of which 2 species recorded for the first time from the state of Himachal Pradesh.

\section{List of taxa \\ Subfamily Syrphinae Tribe Syrphini}

I. Genus Episyrphus Matsumura \& Adachi, 1917

1. Subgenus Episyrphus Matsumura \& Adachi, 1917

1. Episyrphus (Episyrphus) balteatus (De Geer, 1776)

II. Genus Ischiodon Sack, 1913

2. Ischiodon scutellaris (Fabricius, 1805)

III. Genus Sphaerophoria Lepeletier \& Serville, 1828 
Int J Adv Life Sci Res. Volume 2(4)21-37

$>$ Subgenus Knutsonia Barkalov, 2012

3. Sphaerophoria (Knutsonia) viridaenea Brunetti, 1915

IV. Genus Syrphus Fabricius, 1775

> Subgenus Syrphus Fabricius, 1775

4. Syrphus(Syrphus) torvus Osten Sacken, 1875

5. Syrphus (Syrphus) vitripennis Meigen, $1822^{* *}$
V. Genus Chrysotoxum Meigen, 1800

6. Chrysotoxum convexam Brunetti, 1915

2. Tribe Bacchini

VI. Genus Melanostoma Schiner, 1860

7. Melanostoma orientale (Wiedemann, 1824)

http://www.publications.gc.ca/pub?id=9.610315\&sl=0

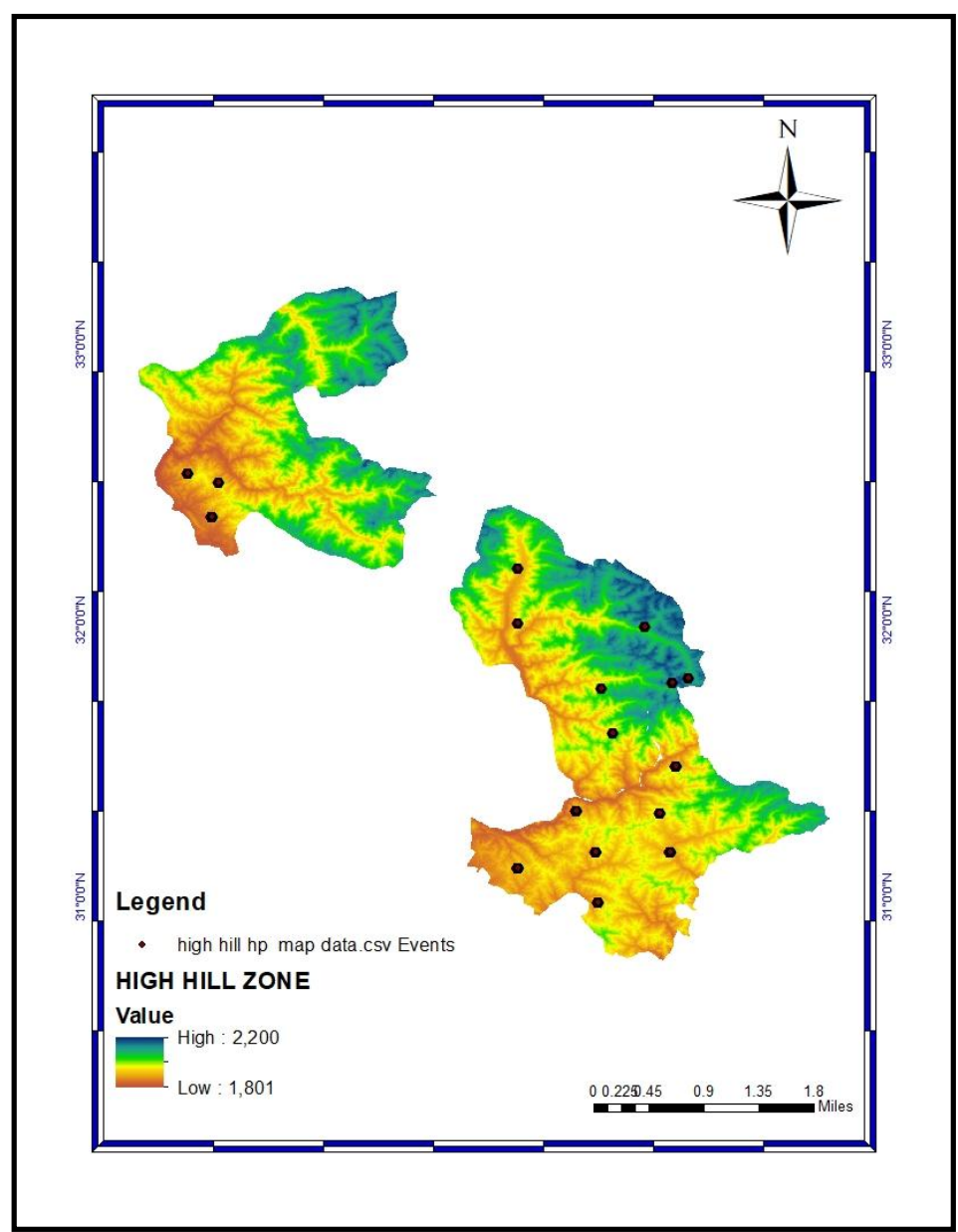

Figure 1: 3D Map showing study area and syrphid species richness from High hill zone from the state of Himachal Pradesh. 
Int J Adv Life Sci Res. Volume 2(4)21-37

Sengupta et. al.

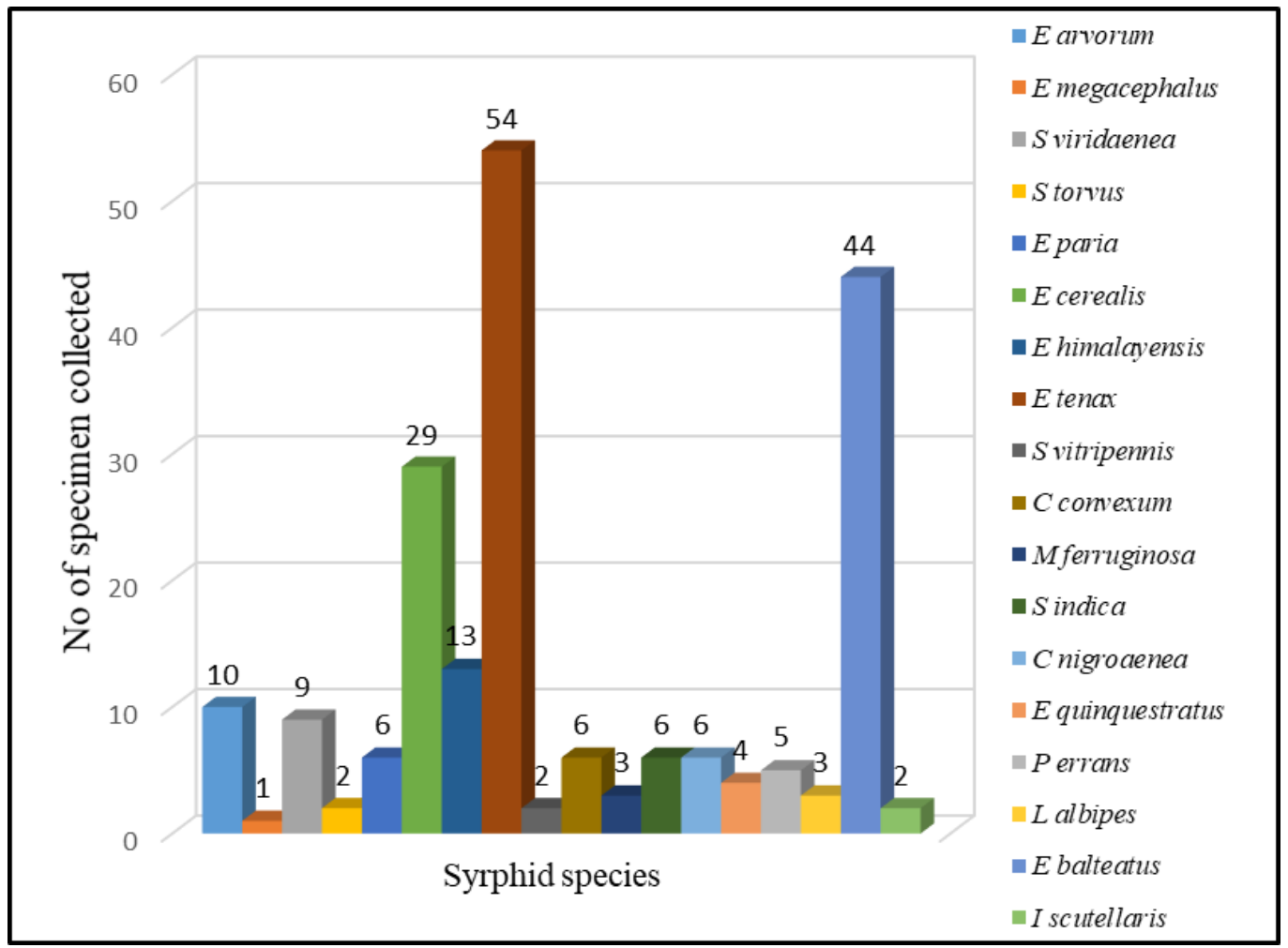

Figure 2: Abundance status of syrphid species from High hill Zone, Himachal Pradesh

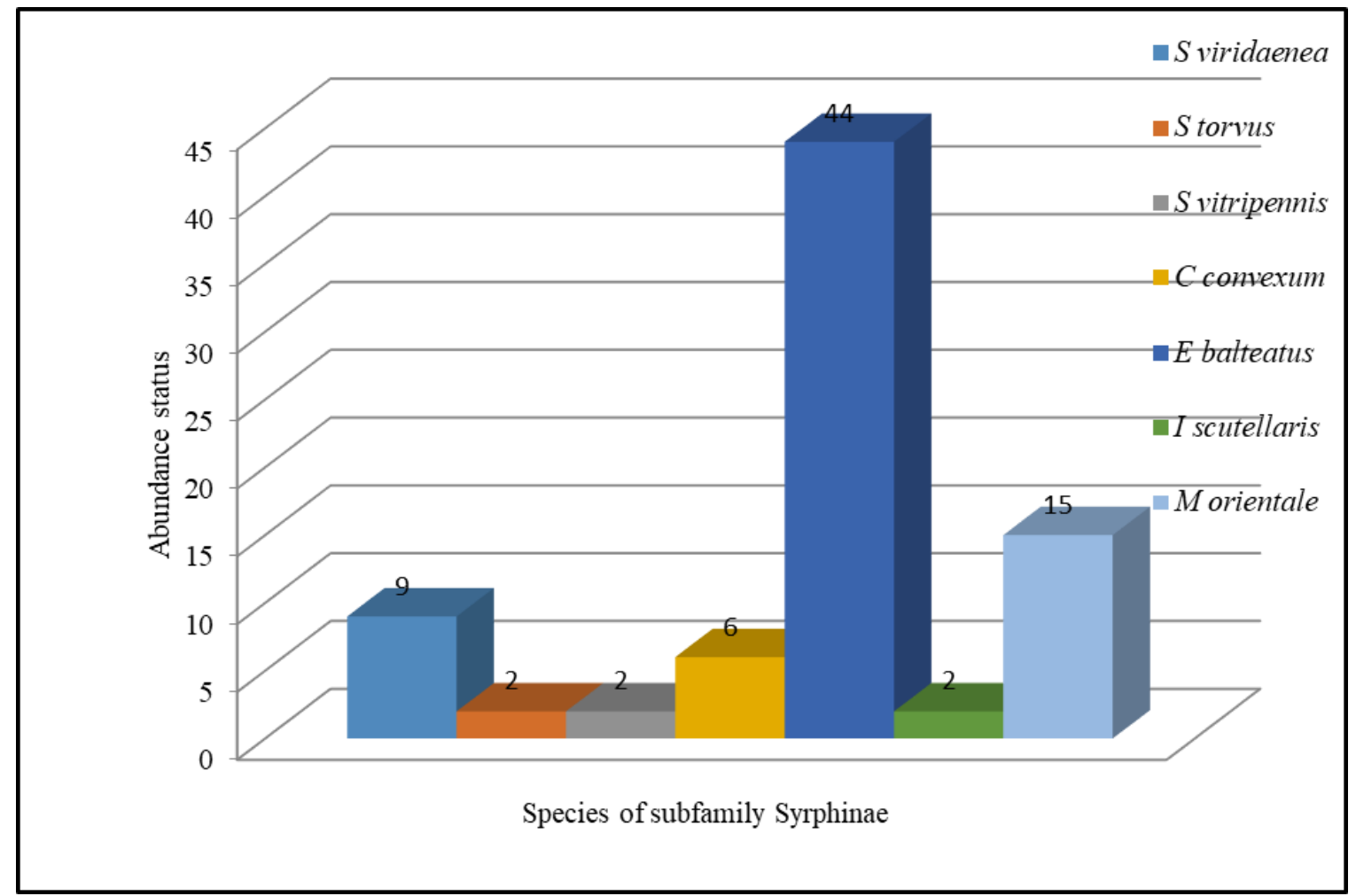

Figure 3: Abundance status of species belonging to Syrphinae subfamily from High hill Zone, Himachal Pradesh 


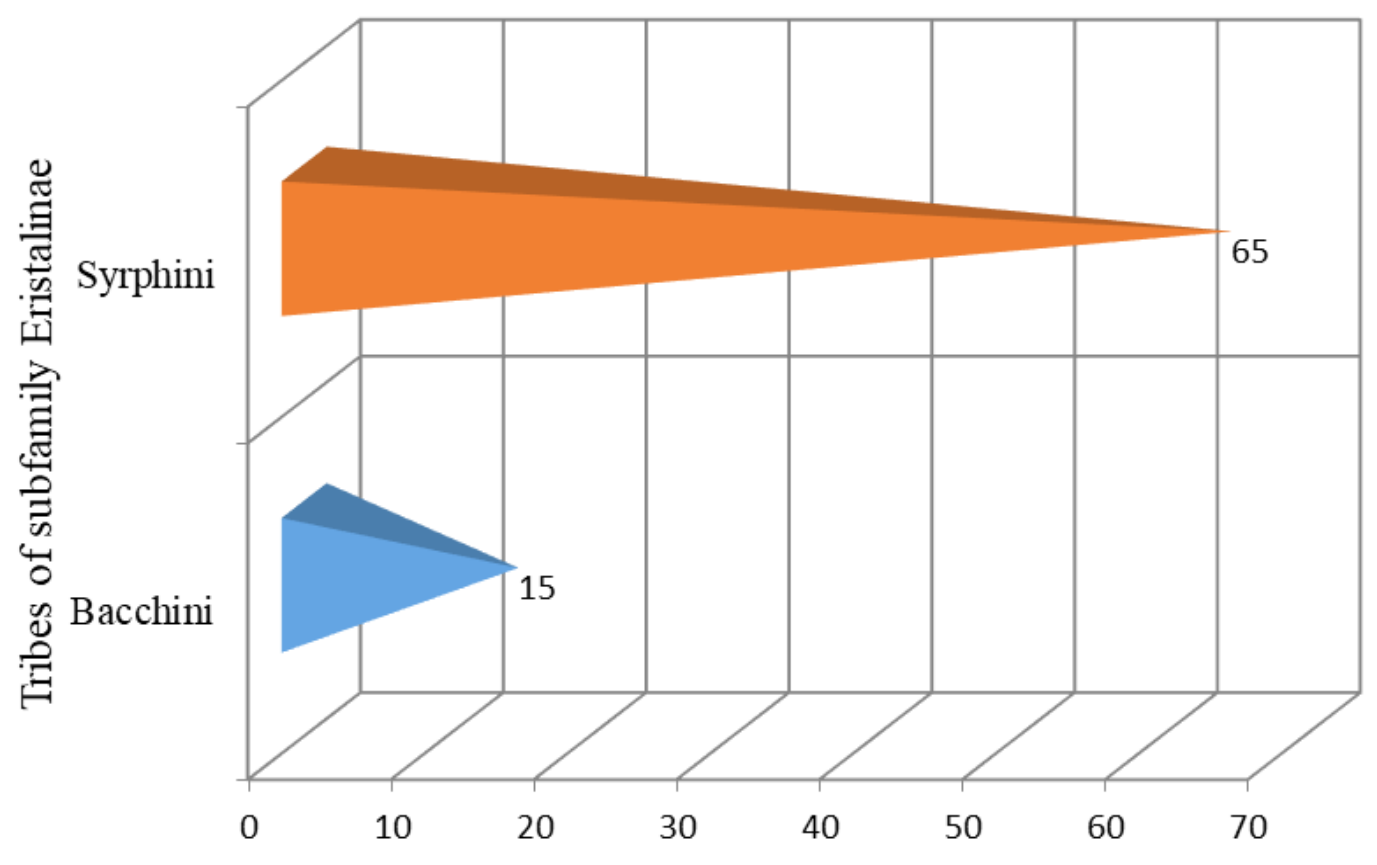

Abundance status of tribes under Eristalinae subfamily

Figure 4: Abundance status of different tribes under Syrphinae subfamily from High hill Zone, Himachal Pradesh

\section{Subfamily Eristalinae}

Tribe Rhingiini

VII. Genus Cheilosia Meigen, 1822

8. Cheilosia nigroaenea Brunetti, 1915

\section{Tribe Eristalini}

VIII. Genus Eristalinus Rondani, 1845

Subgenus Eristalinus Rondani, 1845

9. Eristalinus (Eristalinus) arvorum (Fabricius, 1787) **

10. Eristalinus (Eristalinus) megacephalus (Rossi, 1794)

11. Eristalinus(Eristalinus) quinquestriatus (Fabricius, 1794)

$>$ Subgenus Eristalodes Mik, 1897

12. Eristalinus (Eristalodes) paria (Bigot, 1880)

IX. Genus Eristalis Latreille, 1804

Subgenus Eoseristalis Kanervo, 1938

13. Eristalis (Eoseristalis) cerealis Fabricius, 1805
14. Eristalis (Eoseristalis)himalayensis Brunetti, 1908

Subgenus EristalisLatreille, 1804

15. Eristalis (Eristalis) tenax (Linnaeus, 1758) **

X. Genus Phytomia Guerin-Meneville, 1833

Subgenus Phytomia Guerin-Meneville, 1833

16. Phytomia (Phytomia) errans (Fabricius, 1787)

XI. Genus Lycastris Walker, 1857

17. Lycastris albipes Walker, 1857

XII. Genus Milesia Latreille, 1804

18. Milesia ferruginosa Brunetti, 1913

\section{Tribe Milesiini}

XIII. Genus Syritta Lepeletier \& Serville, 1828

19. Syritta indica (Wiedemann,1824)

*** New record of hoverfly species from state of Himachal Pradesh 


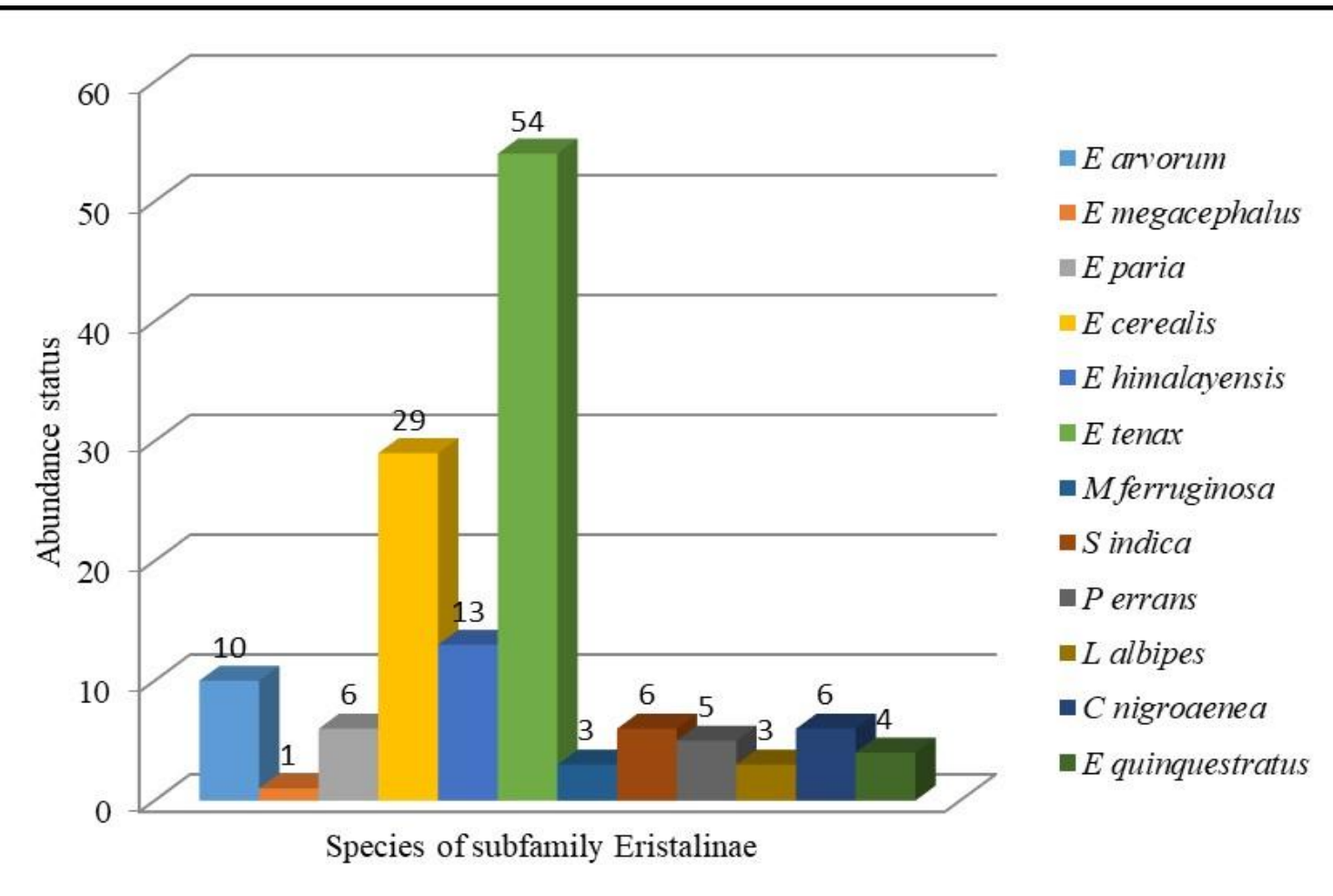

Figure 5: Abundance status of species belonging to Eristalinae subfamily from High hill zone, Himachal Parades

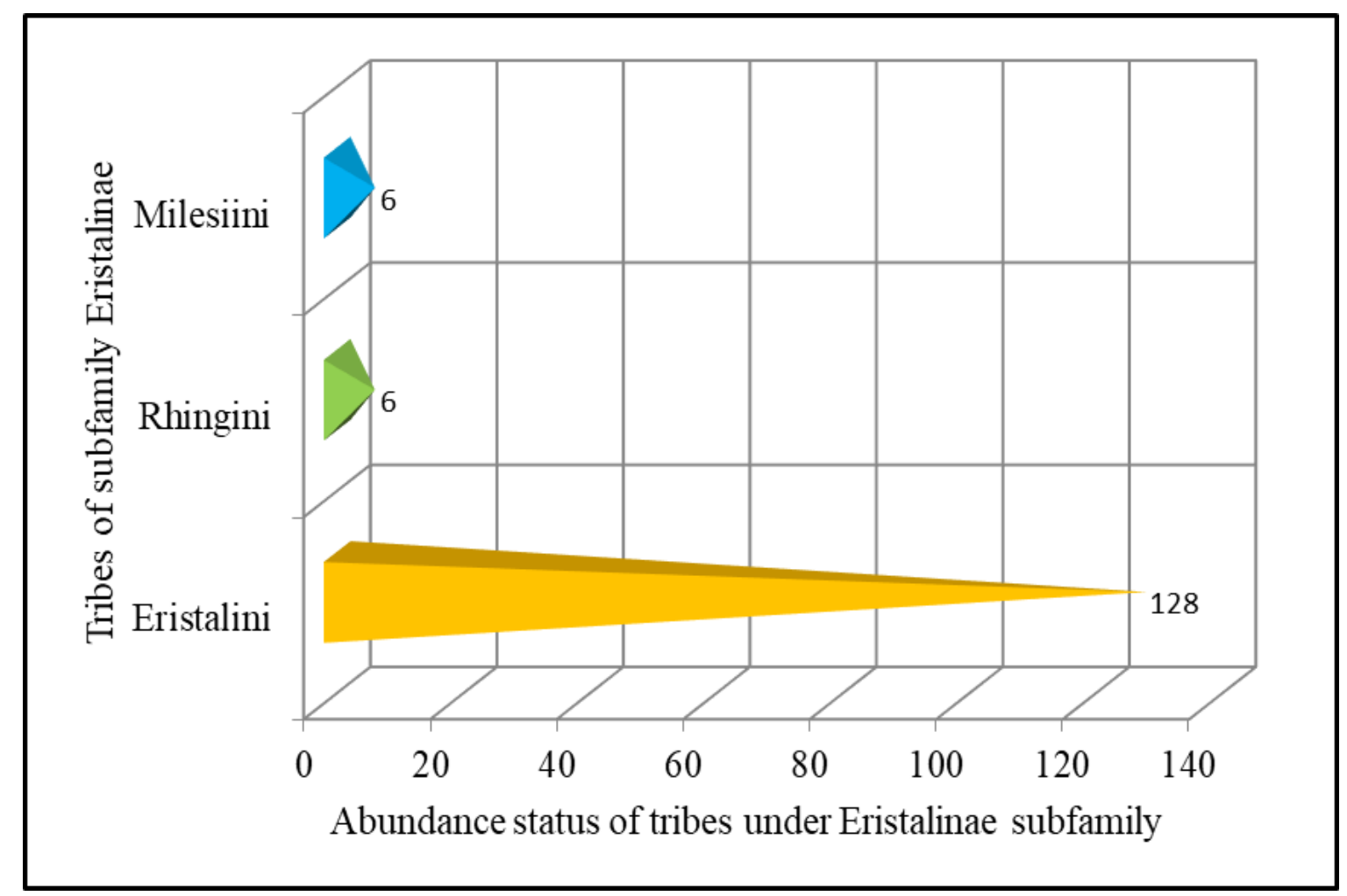

Figure 6: Abundance status of different tribes under Eristalinae subfamily from High hill Zone, Himachal Pradesh 


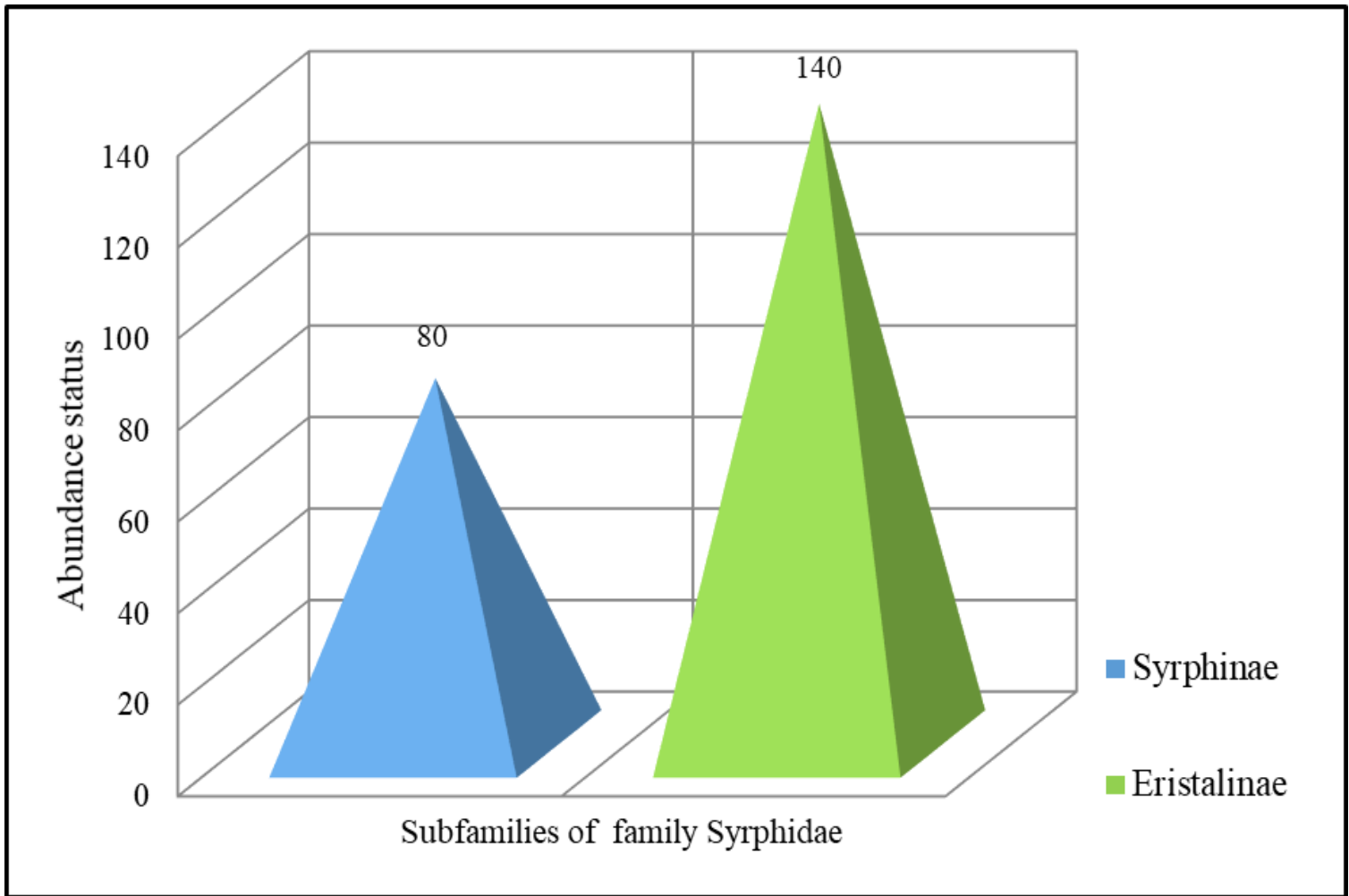

Figure 7: Comparative account of Abundance status of two subfamilies from High hill Zone, Himachal Pradesh.

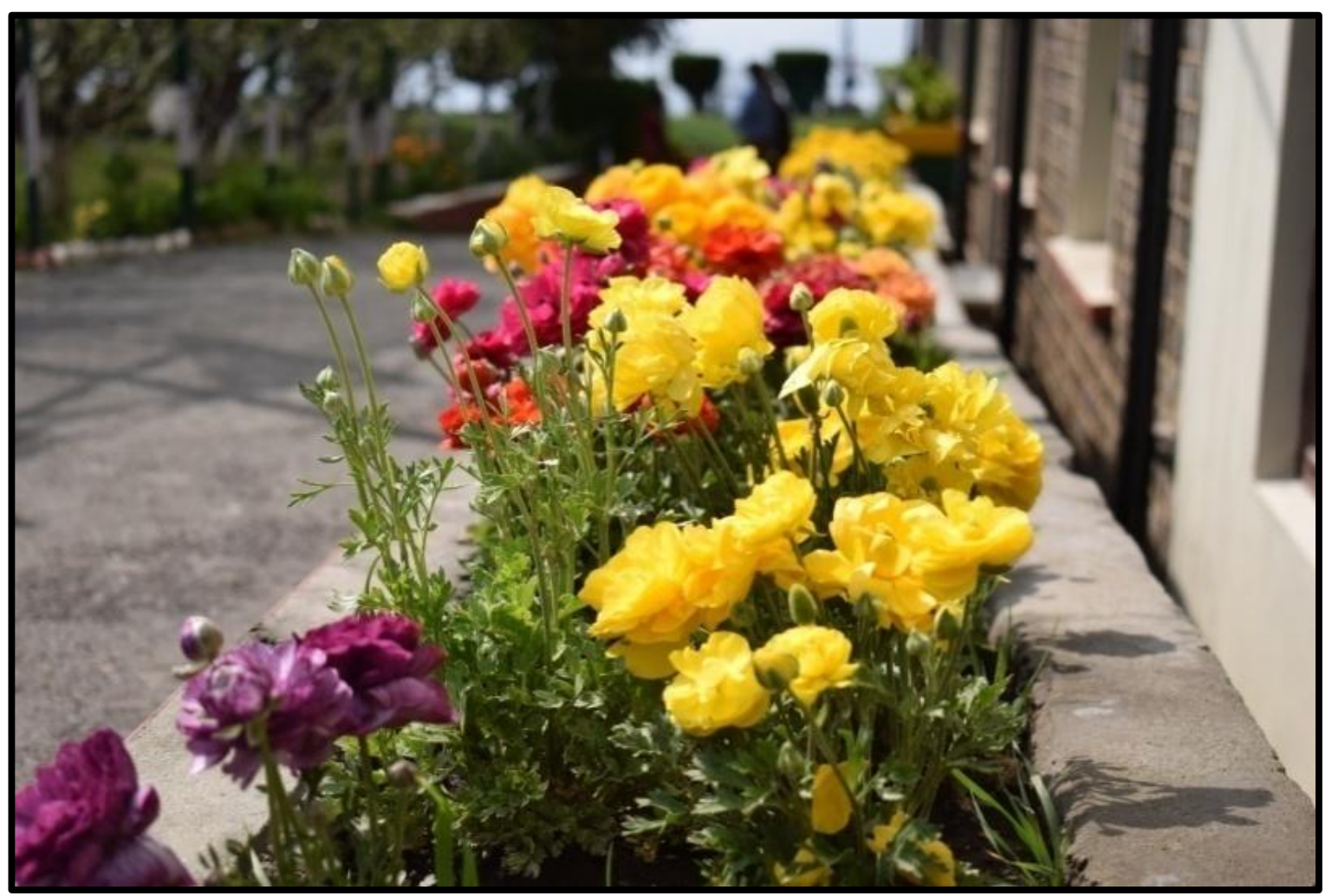

Figure 8: Habitat of hoverflies from High Hill zone of Himachal Pradesh 


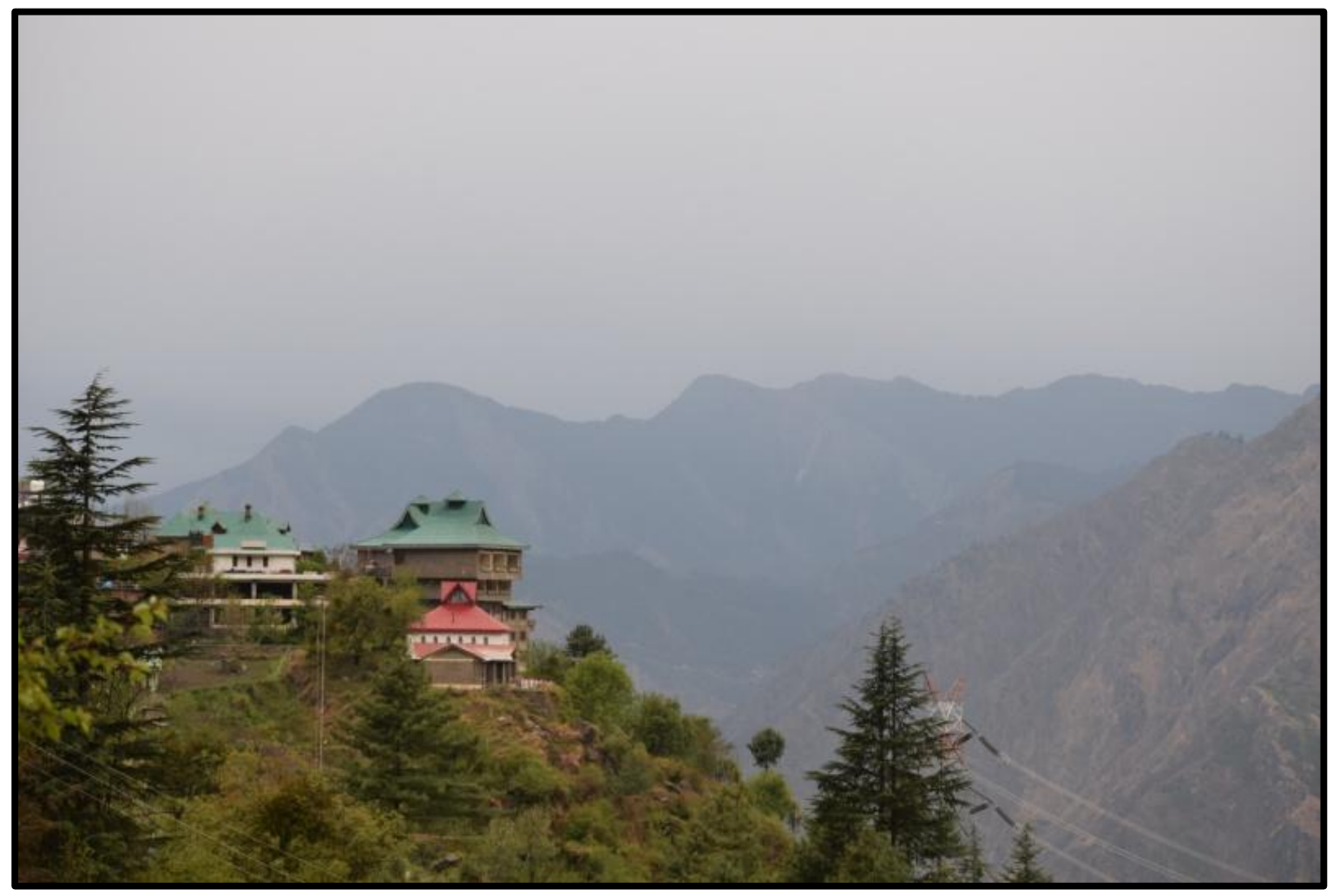

Figure 9: Upper elevation Landscape from High Hill zone of Himachal Pradesh

Key to sub families, tribe, genera and species of hover flies from the High Hill zone of Himachal Pradesh.

\section{Systematic account}

Family Syrphidae

Key to subfamily

1. Post pronotum bare, male abdominal $5^{\text {th }}$ tergite visible in dorsal view. Syrphinae

- Post pronotum with at least a few suberrect or appressed hairs, male abdominal $5^{\text {th }}$ tergitenot visible in dorsal view...Eristalinae

Subfamily Syrphinae

Key to tribe

1. Abdomen usually slender in appearance.

Bacchini

- Abdomen usually equilateral in appearance.

Syrphini
Tribe SYRPHINI

Key to the Genera

1. Antennae elongate, may be longer than head; scape and pedicel often longer than broad; abdomen strongly convex dorsally and margined......... Chrysotoxum Meigen, 1800

- Antennae shorter than head; scape and pedicel not longer than broad; abdomen variable, but posterolateral angles of tergites without projecting. ..2

2. Calypter with lower lobe pilose; Antennae short, 1st flagellomeres twice as long as wide; abdomen without posterolateral angles of tergites projecting, lower lobe of calypter pilose above, especially on posteromedial portion.................Syrphus Fabricius, 1775

- Calypter bare. .3

3. Anterior anepisternum bare. Ischiodon Sack, 1913 
- Anterior anepisternum pilose at least

posterodorsally.

Episyrphus Matsumura \& Adachi, 1917

Genus SyrphusFabricius, 1775

Key to species

1.hind tibia with a row of black bristles below outer side. torvus Osten Sacken, 1875

- hind tibia with golden yellow bristles on inner side. vitripennis Meigen, 1822

Subfamily Eristalinae

Key to tribe

Tribe Eristalini

Key to the genera

2. Eyes bare; submarginal cell of normal length; metathoracic spiracular pile patch present...Phytomyia Guerin-Meneville, 1833

Eyes at least partially pilose, if bare then submarginal cell narrowed, basally due to a r-m cross vein; metathoracic spiracular patch usually absent.

.3

3. Katepiternum bare, eyes densely hairy with two vertical ands of longer hairs; antennal arista with short hairs on basal half.

Eristalis Latreille, 1804

Katepiternum haired; antennal arista practically bare; eyes with dark spots; post alar Ridge with tuft of strong black hairs..................Eristalinus Rondani, 1845

Genus Eristalis Latreille, 1804

Key to the subgenera

1. Eyes bare in appearance.

Eoseristalis Kanervo, 1938

- Eyes densely haired with two vertical band of longer hairs

Eristalis Latreille, 1804

Key to the species of subgenus Eoseristalis Kanervo, 1938

1. Abdominal tergite 3 within male with a triangular or oblique spot on anterior margin reaching laterally in female spot smaller, narrower in the same position.

cerealis Fabricius, 1805
- Abdominal tergite with such triangular spot.

himalayensis Brunetti, 1908

Genus Eristalinus Rondani, 1845

Key to the subgenera

1. Metathoracic spiracle dorsally with a distinct patch of hairs; eyes with longitudinal dark stripes

Eristalodes Mik, 1897

- Metathoracic spiracle infront without any patch of hairs; eyes with spots or irregular markings.

Eristalinus Rondani, 1845

Subgenus Eristalinus Rondani, 1845

Key to the species

1. Scutum with 5 distinct black longitudinal dust bands........

megacephalus (Rossi, 1794)

3. - Scutum with 4 distinct black longitudinal bands..................2

4. 2. Femora normally all orange or brownish orange; fore and mid tibia on apical half; hind pair whollyexcept narrowly at base blackish.

arvorum (Fabricius, 1787)

5. - Femora black; tips generally more or less pale.

quinquestriatus (Fabricius, 1794)

Tribe MILESSINI

Key to genera

1. Marginal cell $(\mathrm{R} 2+3)$ closed before wing margin; cross vein $r$ $\mathrm{m}$ always oblique; usually beyond middle of discal cell $(\mathrm{dm}) \ldots \ldots$... Milesia Latreille, 1804

6. - Marginal cell $(R 2+3)$ open to wing margin .2

2. Epistome conspicuously produced, snout-like; very densely pubescent species; proboscis slender and highly produced; twice as long as epistome; ventrum conspicuously. 
Abnormal........Lycastris Walker, 1857

- Epistome not conspicuously produced, never snout-like, face not more than normally produced at upper mouth edge; comparatively bare species... Syritta Lepeletier \& Serville, 1828

1. Episyrphus (Episyrphus) balteatus (De Geer, 1776)

1776. Musca balteata De Geer, Mem. pour. serv. Hist. Ins.6: 116

Type locality: Sweden

Material examined:7우우 Khajjiar, Chambadistrict, $\quad 1809 \mathrm{mt}$, 32³3'20.8"N,76³'56.09"E, 04.vii.15, coll: J. Sengupta, 8 우 Shimla, Shimla district, 2100mt, $\quad 31^{\circ} 6^{\prime} 16.5^{\prime \prime} \mathrm{N}, 77^{\circ} 10^{\prime} 24.3^{\prime \prime} \mathrm{E}$, 18.iv.17, coll: J.Sengupta.9 ${ }^{\lambda}{ }^{\lambda} \mathrm{Kothi}$, Shimla district, $2408 \mathrm{mt}, 31^{\circ} 28^{\prime} 21.46 " \mathrm{~N}$, 77044'36.79"E, 18.iv.17, coll: J.Sengupta.9우 $7 \delta^{\AA}$ Shimla, Shimla district, 2100mt, 31ㅇ' $16.5^{\prime \prime} \mathrm{N}, 77^{\circ} 10^{\prime} 24.3^{\prime \prime} \mathrm{E}$, 18.iv.17, coll: J.Sengupta, $4{ }^{\lambda} \partial^{7}$ Kullu, Kullu district, 2195mt, 31 $59^{\prime} 16.7994 " \mathrm{~N}, 77^{\circ} 10^{\prime}$ 19.1994"E, 28.iv.18,coll: J.Sengupta.

Distribution: India: Himachal Pradesh, West Bengal.

Distribution: elsewhere: Australasian Region (Australia), Oriental Region (Widely distributed), Palearctic Region (England).

Remarks: The main noticeable remarks regarding this species are that in spite of changes in elevation gradient and habitat types, this species is found to be most abundant.

2. Ischiodon scutellaris (Fabricius, 1805)

1805. Scaeva scutellarisFabricius, Syst. Antliat.: 252

Type locality: India: West Bengal.

Material examined: $1 \widehat{\partial} \delta^{\lambda}$ Dasholi, Shimla district, $2421 \mathrm{mt}, 31^{\circ} 230.38^{\prime \prime} \mathrm{N}$, 77035'41.18"E, 18.iv.17, coll: J.

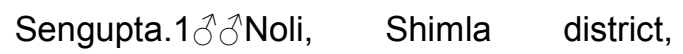

$2004 \mathrm{mt}$, $30^{\circ} 58^{\prime} 45.79 " \mathrm{~N}$, 77²7'42.42"E18.iv.17, coll: J.Sengupta.

Distribution: India: Himachal Pradesh, Andhra Pradesh, Assam, Dellhi, Jammu \&Kashmir, Karnataka, Kerala, Meghalaya, Madhya Pradesh, Manipur, Meghalaya, Orissa, Punjab, Tamil Nadu, Tripura, and West Bengal)

Distribution: Elsewhere: Australasian Region (Australia, Pacific island), Oriental Region (Indonesia, Java), Palaearctic Region (Japan).

Remarks: This species is not so much abundant in this high hill zone area which may be affected by thefact of higher altitudinal landscape.

3. Sphaerophoria(Knutsonia) viridaenea Brunetti, 1915

1915. Sphaerophoria viridaenea Brunetti, Rec. Ind. Mus.11:216

Type locality: Simla, Himachal Pradesh.

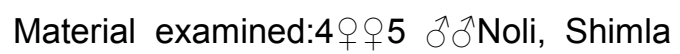
district, $\quad 2004 \mathrm{mt}, \quad 30^{\circ} 58^{\prime} 45.79 " \mathrm{~N}$, 77²7'42.42"E18.iv.17, coll: J. Sengupta.

Distribution: India: Himachal Pradesh, Jammu \& Kashmir, Uttarakhand.

Distribution: Elsewhere: Oriental Region (Afghanistan, Pakistan, Nepal).

Remarks: These species are moderately abundant in this high hill zone area.

\section{Syrphus(Syrphus)torvus OstenSacken, 1875}

1875. Syrphus torvus Osten Sacken, Pros. Boston Soc. Nat. Hist.18:139

Type locality: Canada; Colorado, N.H., R.I., USA

Material examined:2 $q+$ Shimla, Shimla district, $2100 \mathrm{mt}, 31^{\circ} 6^{\prime} 16.5^{\prime \prime} \mathrm{N}, 77^{\circ} 10^{\prime} 24.3^{\prime \prime} \mathrm{E}$, 18.iv.17, coll: J. Sengupta.

Distribution: India: Himachal Pradesh, Jammu \& Kashmir, Uttarakhand.

Distribution: Elsewhere: Nearctic Region (Alaska, Green land, North Carolina, New 
Mexico), Oriental Region (Nepal, Pakistan).

Remarks: This species are rare in distribution in study area

\section{Syrphus (Syrphus) vitripennis Meigen, $1822^{\star *}$}

1822. Syrphus vitripennis Meigen, europ.zwiefl. Insekten. 10:308

Type locality: Germany.

Material examined: $2 q q$ Khajjiar, Chamba district, $1809 \mathrm{mt}$, $32^{\circ} 33^{\prime} 20.8^{\prime \prime} \mathrm{N}, 76^{\circ} 3^{\prime} 56.09 " \mathrm{E}$, 04.vii.15, coll: J.Sengupta

Diagnosis: Epicranium dark, densely yellow-gray pruinose on upper half in male, where as in female the upper one third of epicranium is shining black. Antennal sockets yellow in colour. Both gene and sub gene region yellow. Wing axillary membrane with extensive bare areas. Fore and mid femora black on basal one-third, otherwise yellow, with yellow hairs on posterior surface; Abdominal $3^{\text {rd }}$ and $4^{\text {th }}$ Tergites with entire yellow bands, broadly and shallowly emarginated posteriorly, reaching lateral margins on about half their length.

Distribution: India: Himachal Pradesh, Arunachal Pradesh, Jammu \& Kashmir, Meghalaya, Sikkim.

Distribution: Elsewhere: Oriental Region (Afghanistan, Mangolia), Palearctic Region (China, Europe, Japan, Russia, Siberia), Nearctic Region (Canada).

Remarks: This species is reported for the first time from the state of Himachal Pradesh.

\section{Chrysotoxum convexum Brunetti, 1915}

1915. Chrysotoxum convexum Brunetti, Rec. Indian Mus. 11: 249

Type locality: Garhwal District, Andarban

Material examined:3 $3 q 3^{3} \partial^{\top}$ Tandali, Shimla district, $2149 \mathrm{mt}, 31^{\circ} 9^{\prime} 44.46 " \mathrm{~N}$, 77²3'16.87"E, 18.iv.17, coll: J. Sengupta.
Distribution: India: Himachal Pradesh, Jammu \& Kashmir, Mizoram, Uttar Pradesh and Uttarakhand.

Distribution: Elsewhere: Oriental Region (Pakistan), Palearctic region (China)

Remarks: This species was less abundant and habitat specific in study sites.

7. Melanostoma orientale (Wiedemann, 1824)

1824. Syrphus orientalis, Wiedemann, Analec. Ent: 36

Type locality:India: Tamil Nadu: Tharangambi.

Material examined:3 $+q$ Khajjiar, Chamba district, $1809 \mathrm{mt}$, 32³3'20.8"N,76³'56.09"E, 04.vii.15, coll: J. Sengupta, 6 우 우 Shimla, Shimla district, 2100mt, $\quad 31^{\circ} 6^{\prime} 16.5^{\prime \prime} \mathrm{N}, 77^{\circ} 10^{\prime 2} 24.3^{\prime \prime} \mathrm{E}$, 18.iv.17, coll: J. Sengupta. $4 \widehat{\partial}$ Chamor, Shimla district, 1972mt, 31¹8'29.72"N,77²2'57.66"E, 18.iv.17, coll: J. Sengupta, $2 \partial^{\top}$ Ghiaghi, Banjar, Kullu district, 2205mt, $31^{\circ} \quad 4^{\prime}$ 17.9994"N,77²8' 26.4"E, 28.iv.18, coll: J. Sengupta,

Distribution: India: Himachal Pradesh, Assam, Arunachal Pradesh, Jammu \& Kashmir, Karnataka, Meghalaya, Sikkim, Tamil Nadu, Tripura, Uttar Pradesh, and West Bengal.

Distribution: elsewhere: Oriental Region (Bhutan, Nepal, Pakistan, Sri Lanka)

Remarks: This species was quite abundant across the study area

\section{Cheilosia nigroaenea Brunetti, 1915}

1915. Chilosia nigroaenea Brunetti, Rec. Indian Mus.11: 204

Type locality: Matiana, Simla, Himachal Pradesh.

Material examined: $5 \hat{0} 1+$ Noli, Shimla district, $\quad 2004 \mathrm{mt}, \quad 30^{\circ} 58^{\prime} 45.79^{\prime \prime} \mathrm{N}$, 77²7'42.42"E18.iv.17, coll: J. Sengupta.

Distribution: India: Himachal Pradesh, Sikkim.

Distribution: elsewhere: Oriental Region (Nepal) 
Remarks: This hoverfly is unique because of wholly black in appearance which is quite different from the regular yellowblack patterns of syrphds.

\section{Eristalinus (Eristalinus) arvorum (Fabricius, 1787)}

1787. Syrphus arvorum Fabricius, Mantissa insectorum.2(2): 335

Type locality: Tamil Nadu: Tharangambadi.

Material examined: $2 q q$ Tandali, Shimla district, $2149 \mathrm{mt}, \quad 31^{\circ} \quad 9^{\prime} 44.46 " \mathrm{~N}$, 77043'16.87"E, 18.iv.17, coll: J. Sengupta.2우 Khajjiar, Chamba district, 1809mt, $\quad 32^{\circ} 33^{\prime} 20.8^{\prime \prime} \mathrm{N}, 76^{\circ} 3^{\prime} 56.09 " \mathrm{E}$,

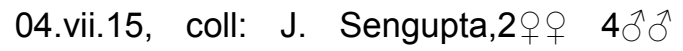
Chamor, Shimla district, $1972 \mathrm{mt}$, 31'18'29.72"N,77²2'57.66"E, 18.iv.17, coll: J. Sengupta.

Diagnosis: Ommatidium on upper part of eyes are much larger than on lower half.eyes bear with minute dots especially in lower part. Vertical tubercle small and blackish. All of the Epicranium, genae and sub genae region covered with yellowish grey tomentum. Post cranium area grey with small yellowish pubescences, fronto genal sutures covered with yellowish grey hairs. Base of flagellomere darker with carrotish bare arista. Meso notum and meso pleuron covered with yellowish grey dust. Presence of 4 equidistant shining black stripes on dorsum area of mesonotum. scuto scutellar suture brownish yellow; Katepimeron with grayish yellow pubescences. Abdominal coloration mainly black. 2 large oblong yellow spots on $2^{\text {nd }}$ abdominal tegites. Pair of diamond shaped yellow spot on $3^{\text {rd }}$ abdominal segment. Abdominal tergites covered with ground coloured pubescences. brownish yellow to carrotish in appearance. Tarsi all orange, trochanter and basal tibia is blackish in appearance. Both stalk and blade area of wing are clear, a small brownish black spot at each end of costa. Halteres ochre yellow in colour. Length:10$12 \mathrm{~mm}$.

Distribution: India: Himachal Pradesh, Arunachal Pradesh, Jammu \& Kashmir,
Manipur, Meghalaya, Mizoram, Orissa, Sikkim, Tripura, West Bengal.

Distribution: Elsewhere: Afro tropical region (Seychelles), Australasian region(Australia), Oriental Region (Entire SE Asia), Indo Australian region (Hawaii, Micronesia), Palearctic region (China, Japan Mariana)

Remarks: This species is a new record from High hill zone as well as from the state of Himachal Pradesh.

10. Eristalinus (Eristalinus) megacephalus (Rossi, 1794)

1794. Syrphus megacephalus Rossi, Mantissa insectorum.2: 63

Type locality: Toscana, Italy.

Material examined:1옹 Shimla, Shimla district, $2100 \mathrm{mt}, 31^{\circ} 6^{\prime} 16.5^{\prime \prime} \mathrm{N}, 77^{\circ} 10^{\prime} 24.3^{\prime \prime} \mathrm{E}$, 18.iv.17, coll: J. Sengupta. (check from diversity)

Distribution: India: Himachal Pradesh, Andhra Pradesh, Bihar, Gujarat, Jammu \&Kashmir, Karnataka, Kerala, Maharashtra, Meghalaya, Orissa, Rajasthan, Sikkim, Tamil Nadu, Tripura, Uttarakhand, West Bengal.

Distribution: Elsewhere: Indo Australian region (Singapore), Oriental Region (Afghanistan, Buru, Nepal, Pakistan), Palearctic region (China, Japan)

Remarks: This species is distinguished by narrow conical abdomen along with uniformly dotted eyes.

11. Eristalinus (Eristalinus) quinquestriatus (Fabricius, 1794)

1794. Syrphus quinquestriatus Fabricius, Entomol. Syst. Emendat. et auct...4(6):289

Type locality: Tharangambadi, Tamil Nadu.

Material examined: $1 \hat{\jmath}$ Chamor, Shimla district, $1972 \mathrm{mt}$, 3118'29.72"N,77²2'57.66"E, 18.iv.17, coll: J. Sengupta.

Distribution: India: Himachal Pradesh, Arunachal Pradesh, Assam, Bihar, Karnataka, Kerala, Madhya Pradesh, 
Meghalaya, Nagaland, Orissa, Tamil Nadu, Tripura, Uttarakhand, West Bengal.

Distribution: Elsewhere: Oriental Region (Buru), Palearctic region (China, Japan)

Remarks: This species is distinguished by narrow conical abdomen along with uniformly dotted eyes.

\section{Eristalinus (Eristalodes) paria (Bigot, 1880)}

1880. Eristalomyia paria Bigot, Ann. Soc. Ent. Fr. ser. 5, 10: 218

Type locality: Sri Lanka.

Material examined: $4 ㅇ$ Tandali, Shimla district, $2149 \mathrm{mt}, \quad 31^{\circ} \quad 9^{\prime} 44.46 " \mathrm{~N}$, 77043'16.87"E, 18.iv.17, coll: J. Sengupta,2우 Shimla, Shimla district, 2100mt, $\quad 31^{\circ} 6^{\prime} 16.5^{\prime \prime} \mathrm{N}, 77^{\circ} 10^{\prime} 24.3^{\prime \prime} \mathrm{E}$, 18.iv.17, coll: J. Sengupta.

Distribution: India: Himachal Pradesh, Arunachal Pradesh, Chandigarh, Jammu \& Kashmir Karnataka, Manipur, Meghalaya, Mizoram, Sikkim, Tamil Nadu, Uttarakhand, Uttar Pradesh, West Bengal.

Distribution: elsewhere: Oriental Region (Sri Lanka, Taiwan), Indo-Australian Region (Java, Moluccas)

Remarks: This species of hoverfly can be easily distinguished by greenish striped pattern in both of the eyes in both gender.

\section{Eristalis (Eoseristalis) cerealis Fabricius, 1805}

1805. Eristalis cerealis Fabricius, Syst. Antliat.14: 232

1880. Eristalis barbata Bigot, Ann. Soc. Ent. Fr. ser.5, 10:214

Type locality: China.

Material examined:2 $20+11 \widehat{\jmath}$ Khajjiar, Chamba district, 1809mt, 32³3'20.8"N,76³'56.09"E, 04.vii.15, coll: J. Sengupta, $2 \widehat{\partial} \delta^{\AA}$ Chamor, Shimla district, 1972mt, $\quad 31^{\circ} 18^{\prime} 29.72 " N, 77^{\circ} 22^{\prime} 57.66 " \mathrm{E}$, 18.iv.17, coll: J. Sengupta, $1{ }^{1} \mathrm{~K}$ Kothi, Shimla district, $2408 \mathrm{mt}, 31^{\circ} 28^{\prime} 21.46 " \mathrm{~N}$, 77044'36.79"E, 18.iv.17, coll: J. Sengupta. $6 \hat{0} \hat{\sigma}$ Kullu, Kullu district, 2908 $\mathrm{mt}, \quad 31^{\circ} \quad 47^{\prime} \quad 31.2^{\prime \prime} \mathrm{N}, 77^{\circ} \quad 47^{\prime} \quad 20.4^{\prime \prime} \mathrm{E}$, 28.iv.18, coll: J. Sengupta. $7 q+$ Ghiaghi Tindar, Banjar, Kullu district, $2125 \mathrm{mt}, 31^{\circ}$ 46' 22.7994"N,77 43' 55.1994"E,28.iv.18, coll: J. Sengupta,

Distribution: India: Himachal Pradesh, Assam, Jammu \& Kashmir, Meghalaya, Sikkim, Tamil Nadu, West Bengal.

Distribution: elsewhere: Widespread in Oriental region, Palearctic region (Russia)

Remarks: This species is moderately abundant throughout different habitat of this high hill zone.

14. Eristalis (Eoseristalis) himalayensis Brunetti, 1908

1908. Eristalis himalayensis Brunetti, Rec. Indian Mus.2:70

Type locality: Indostan

Material examined: 1 S Shimla, Shimla district, $2100 \mathrm{mt}, 31^{\circ} 6^{\prime} 16.5^{\prime \prime} \mathrm{N}, 77^{\circ} 10^{\prime} 24.3^{\prime \prime} \mathrm{E}$, 18.iv.17, coll: J. Sengupta.1우 Bishog, Shimla district, $2004 \mathrm{mt}$, $30^{\circ} 58^{\prime} 45.79 " N, 77^{\circ} 27^{\prime} 42.42 " \mathrm{E}, \quad$ 18.iv.17, coll: J. Sengupta.1우 Jangal LakhotiKujwi, Chaupal, Shimla district, $2421 \mathrm{mt}, 31^{\circ}$ 2'30.38"N, 77³5'41.18"E, 18.iv.17, coll: J. Sengupta.1우 Bishog, Shimla district, 1963 $\mathrm{mt}, \quad 31^{\circ} \quad 9^{\prime} 35.22 " \mathrm{~N}, \quad 77^{\circ} 27^{\prime} 14.84^{\prime \prime} \mathrm{E}$, 18.iv.17, coll: J. Sengupta. $1 \delta^{\Uparrow}$ Dasholi, Shimla district, $2421 \mathrm{mt}, 31^{\circ} 2 ' 30.38 " \mathrm{~N}$, 77³5'41.18"E, 18.iv.17, coll: J. Sengupta. $10^{\lambda}$ Guthan, Shimla district, $1963 \mathrm{mt}, 31^{\circ}$ 9'35.22"N, 77²7'14.84"E, 18.iv.17, coll: J. Sengupta, 1 Shimla, Shimla district, 2100 $\mathrm{mt}, \quad 31^{\circ} 6^{\prime} 16.5^{\prime \prime} \mathrm{N}, 77^{\circ} 10^{\prime} 24.3^{\prime \prime} \mathrm{E}, \quad 18 . i v .17$, coll: J. Sengupta. $1 \sigma^{\lambda}$ Guthan, Shimla district, $1963 \mathrm{mt}, 31^{\circ} 9^{\prime} 35.22 " \mathrm{~N}$, 77027'14.84"E, 18.iv.17, coll: J. Sengupta. $1 \delta$ Jangal LakhotiKujwi, Chaupal, Shimla

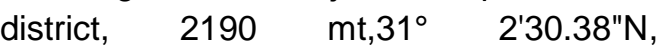
7735'41.18"E, 18.iv.17, coll: J. Sengupta.

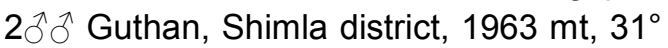
9'35.22"N, 77²7'14.84"E, 18.iv.17, coll: J. Sengupta.1 1 Dalhousie Chamba district, 1985 $32^{\circ} 31^{\prime} 50.21 " \mathrm{~N}, 75^{\circ} 58^{\prime} 46.60 " E, 18$.iv.17, coll: J. Sengupta.1 $\delta^{\lambda}$ Jangal LakhotiKujwi, Chaupal, Shimla district, $2190 \mathrm{mt}, 31^{\circ}$ 2'30.38"N,77³5'41.18"E, 18.iv.17, coll: J. Sengupta, 
Distribution: India: Himachal Pradesh, Arunachal Pradesh, Jammu \& Kashmir, Sikkim, Uttarakhand, West Bengal

Distribution: elsewhere: Oriental Region (China, Java, Myanmar, Nepal Sri Lanka). Indo-Australian Region (Malaya, Philippines, Sumatra, Sumbawa.)

Remarks: This species of hoverfly found to be confined in upper elevational habitats only during study.

15. Eristalis (Eristalis) tenax (Linnaeus, 1758)

1758. Musca tenax Linnaeus, Syst. Nat. Ed. 10: 591

1924. Eristalis claripes Abreu, Mems $R$. Acad. Cienc. Artes. 19(1): 104

Type locality: Europe

Material examined:3우 Khajjiar, Chambadistrict, 1809mt, $32^{\circ} 33^{\prime} 20.8^{\prime \prime} \mathrm{N}, 76^{\circ} 3^{\prime} 56.09 " \mathrm{E}, 04 . v i i .15$, coll: J. Sengupta, 9 우 Shimla, Shimla district, 2100mt, $\quad 31^{\circ} 6^{\prime} 16.5^{\prime \prime} \mathrm{N}, 77^{\circ} 10^{\prime} 24.3^{\prime \prime} \mathrm{E}$, 18.iv.17, coll: J. Sengupta.1우 Mandhol, Shimla district, $1986 \mathrm{mt}, \quad 31^{\circ}$ 8'18.52"N,7741'1.65"E, 19.iv.17, coll: J.Sengupta. $9 \hat{\partial} \sigma^{\lambda} \quad 3$ 우 Kothi, Shimla district, $\quad 2408 \mathrm{mt}, \quad 31^{\circ} 28^{\prime} 21.46 " \mathrm{~N}$, 77044'36.79"E, 18.iv.17, coll: J.Sengupta.2 $q$ Khajjiar, Chamba district, 1809mt, $\quad 32^{\circ} 33^{\prime} 20.8^{\prime \prime} \mathrm{N}, 76^{\circ} 3^{\prime} 56.09 " \mathrm{E}$, 04.vii.15, coll: J.Sengupta, 9 우 $3 \widehat{o}^{3}$ Shimla, Shimla district, $2100 \mathrm{mt}$, $31^{\circ} 6^{\prime} 16.5^{\prime \prime N}, 77^{\circ} 10^{\prime} 24.3^{\prime \prime} \mathrm{E}$, 18.iv.17, coll: J.Sengupta. $4 \hat{\partial} \hat{\sigma}$ Chamor, Shimla district, 1972mt, $\quad 31^{\circ} 18^{\prime 2} 29.72 " N, 77^{\circ} 22^{\prime} 57.66 " \mathrm{E}$, 18.iv.17, coll: J.Sengupta, $1 \hat{0}$ Mandhol, Shimla district, $1986 \mathrm{mt}, \quad 31^{\circ}$ 8'18.52"N,7741'1.65"E18.iv.17, coll:

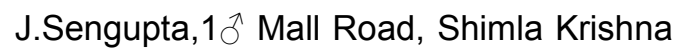
Nagar, Shimla district, $1809 \mathrm{mt}$, $32^{\circ} 33^{\prime} 20.8^{\prime \prime} \mathrm{N}, 7^{\circ} 3^{\prime} 56.09^{\prime \prime E}, 04 . v i i .15$, coll: J.Sengupta, 122 우 Mall Road, Shimla Krishna Nagar, Shimla district,2100 mt, 31'6'16.5"N,77¹0'24.3"E,18.iv.17, coll: J.Sengupta, 132 우 Kullu, Kullu district, $2197 \mathrm{mt}, \quad 31^{\circ} \quad 35^{\prime} \quad 27.6^{\prime \prime} \quad \mathrm{N}, 77^{\circ} \quad 30^{\prime}$ 54"E,28.iv.18, coll:

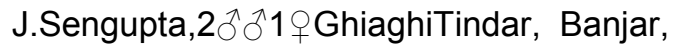

Kullu district,2135 mt,31 $31^{\circ} 27.6^{\prime \prime} \mathrm{N}, 77^{\circ}$ 30' 54"E,28.iv.18, coll: J.Sengupta,

Diagnosis: Ommatidium pubescent with 2 rows of longitudinal bands of brown trichia along the eyes. Epicranium distinctly large, shining black with black and ochre yellow coloured trichia, both sub cranial cavity, genae and sub genae region appear black.scape and pedicle usually brownish black; maxillary part bears bare arista. Meso notum shining brownish black in appearance, scuto scutellar suture brownish yellow; sub scutellar fringe absent, Katepimeron with concolourous pubescences. Abdominal coloration bright carrotish to black. Basal blackish band in both 2nd and 3rd abdominal segments. Venter in both sexes is carrotish yellow in appearance. blackish legs, trochanter and basal 3rdof fore and basal half of middle tibia is ochre yellow in appearance, coxa with yellow coloured macro trichia. Both stalk and blade area of wing are greyish or brownish on anterior half, a small quadrate dark brown spot below the tip of auxillary vein enclosing a small cross vein. Halteres dull brownish yellow in colour. Length:13$15 \mathrm{~mm}$.

Distribution: India: Himachal Pradesh, Arunachal Pradesh, Chandigarh, Jammu \& Kashmir, Manipur, Meghalaya, Mizoram, Nagaland, Punjab, Sikkim, Uttarakhand, West Bengal.

Distribution: elsewhere: Australasian Region (Australia, New Zealand), IndoAustralian Region (Hawaii), Palaearctic Region (China, Japan), Oriental Region (Myanmar, Pakistan, Sri Lanka).

Remarks: This robust species is a new record from High hill zone as well as from the state of Himachal Pradesh.

16. Phytomia (Phytomia) errans (Fabricius, 1787)

1787. Syrphus errans Fabricius, Mantissa insectorum. 2: 337

Type locality: Tamil Nadu: Tharangambadi.

Material examined: $1 \hat{\delta}$ Kothi, Shimla district, $2408 \mathrm{mt}, \quad 31^{\circ} 28^{\prime} 21.46 " \mathrm{~N}$, 
7744'36.79"E, 18.iv.17, coll: J. Sengupta. $1 \hat{\sigma}^{\lambda}$ Chamor, Shimla district, $1972 \mathrm{mt}$, 31'18'29.72"N,77²2'57.66"E, 18.iv.17,

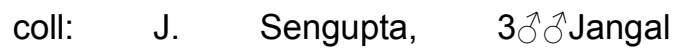
LakhotiKujwi, Chaupal, Shimla district, $2190 \mathrm{mt}, \quad 31^{\circ} \quad 2^{\prime} 30.38^{\prime \prime} \mathrm{N}, 77^{\circ} 35^{\prime} 41.18^{\prime \prime} \mathrm{E}$, 18.iv.17, coll: J. Sengupta,

Distribution: India: Himachal Pradesh, Arunachal Pradesh, Chandigarh, Jammu \& Kashmir, Manipur, Meghalaya, Mizoram, Nagaland, Punjab, Sikkim, Uttarakhand, West Bengal.

Distribution: elsewhere: Australasian Region (Australia, New Zealand), Oriental Region (Myanmar, Pakistan, Sri Lanka). Palaearctic Region (China, Japan),

Remarks: This species is comparatively robust and also appears to be dark purple in colour which is different from normal syrphid's appearance.

\section{Lycastris albipes Walker, 1857}

1857. Lycastris albipes Walker, Trans. Ent. Soc. Lond. 4: 155

Type locality: Hindostan

Material examined:2 $2+10^{\wedge}$ Shimla, Shimla district, 2100mt, $31^{\circ} 6^{\prime} 16.5^{\prime \prime} \mathrm{N}, 77^{\circ} 10^{\prime} 24.3 " \mathrm{E}$, 18.iv.17, coll: J. Sengupta.

Distribution: India: Himachal Pradesh, Uttarakhand, West Bengal.

Distribution: elsewhere: Nil.

Remarks: This species have shown limited availability during collection at study areas. Apart from this, this species is also endemic to the state of Himachal Pradesh.

\section{Milesia ferruginosa Brunetti, 1913}

1913. Milesia ferruginosa Brunetti, Rec. Indian Mus. 9: 268

Type locality: Kumaon district, Darjeeling

Material examined:2 $\delta^{\top}{ }^{\top}$ Tandali, Shimla district, $2149 \mathrm{mt}, \quad 31^{\circ} \quad 9^{\prime} 44.46 " \mathrm{~N}$, 7743'16.87"E, 18.iv.17, coll: J. Sengupta, $1{ }^{\top}$ Kothi, Shimla district,

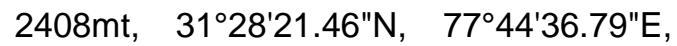
18.iv.17, coll: J. Sengupta.
Distribution: India: Himachal Pradesh, West Bengal.

Distribution: elsewhere: Oriental Region (Laos)

Remarks: This species is also robust in appearance and has shown limited distribution and abundance.

\section{Syritta indica (Wiedemann, 1824)}

1824. Eumerus indica Wiedemann, Analecta entomologica.: 33

Type locality: East Indies

Material examined:5 51 Dalhousie Chamba district, $1985 \mathrm{mt}, 32^{\circ} 31^{\prime} 50.21^{\prime \prime} \mathrm{N}$, 7558'46.60"E, 18.iv.17, coll: J.Sengupta.

Distribution: India: Himachal Pradesh, Madras, Pondicherry, West Bengal.

Distribution: elsewhere: Oriental Region (Nepal, Taiwan)

Remarks: This species is distinguished by the presence of swollen hind part of legs.

\section{Discussion \& Conclusion}

No doubt that the studies regarding conservation strategies (Ferrier et al;2016) of pollinating groups are usually focused with non-dipteran pollinators only (Sengupta et al.,2019). That's why it has become utmost important to establish the proficiency of pollinating hoverflies especially in higher elevational landscapes. The present studies thus have focused on the availability of one of the major group of this second line pollinating flies from High hill zone of Himachal Pradesh. Altogether 19 species under 13 genera over 2 subfamilies have been reported from this zone (figure 2). Among subfamilies Syrphinae $(36.36 \%)$ has shown a higher abundance percentile value than Eristalinae (63.63\%) (figure 3,5,7). Further calculation among Syrphinae subfamily has shown that tribe Syrphini exhibited the maximum abundance value with a percentile of $81.25 \%$ while Bacchini has shown the least value (18.75\%) (figure 4). On the other hand, within the Eristalinae subfamily, Eristalini tribe has shown the maximum abundancy value $(91.4 \%)$ followed by Milesini (4.28\%) and 
Rhingini (4.28\%) (figure 6). Onthe other hand among the reported species $\mathrm{E}$ tenax found to be most abundant (24.54\%) whereas $E$ megacephalas have shown the least abundancy value of $0.45 \%$. while $\mathrm{E}$ balteatus (20\%), E himalayensis (5.90\%), and E cerealis $(13.18 \%)$, has shown moderate value in tem of their abundance. 3 species found to be recorded newly from the state, whereas 1 species found to be endemic to state of Himachal Pradesh. Among 19 species 94.73 \%of total available syrphid has shown large distributional affinity towards Oriental region, $26.31 \%$ of total available syrphid species shown distributional affinity towards both Australian and Indo Australian realms. While $21.05 \%$ of total available syrphid species shows confinement in distribution within Palearctic region and $10.52 \%$ towards Nearctic realm. On the other hand, only $5.26 \%$ of total species available from High hill zone has shown distributional affinity towards Afro Tropical realm. Such a wide distributional range and high index of abundance values of the species in the studied zone have suggested towards a balanced ecosystem health from this region. This will in turn

\section{References}

Banerjee D, Naskar A Sengupta J, Hazra S and Maity A. 2018. Insecta: Diptera. In: Faunal Diversity of Indian Himalaya. (Eds. Chandra, K., Gupta, D., Gopi, K.C., Tripathy, B. and Kumar, V.), Director, Zool. Surv. India. Kolkata, India: Chapter 41 727-760.

Brunetti, E. (1907). Notes on the Oriental Syrphidae. Part I. Records of the Indian Museum, 1, 11-13.

Evenhuis N.L. \& Pape T. (editors). (2019). Systema Dipterorum, Version (2.3). http://www.diptera.dk/, accessed on (15/07/2019).

Ferrier, Simon.,Ninan, K.N.,Leadley, P.,Alkemade, Rob.,Acosta, L.A.,Akcakaya, H. R.,Brotons, L., Cheung, W., Christensen, V., Harhash, K.A.,Kabubo,M. J., Lundquist, C.,Obersteiner, M., Pereira, H., Peterson, G.,Pichs. R.M., Ravindranath, N. H.,Rondinini, C., \&Wintle, B. (2016). IPBES (2016): Summary for policymakers of the methodological assessment of scenarios and models of biodiversity and ecosystem services of the Intergovernmental Science-Policy Platform on Biodiversity and Ecosystem Services.

Ghahari, H., Hayat, R., Tabari, M., \&Ostovan, H. (2008). Hover flies (Diptera: Syrphidae) from rice fields and around grasslands of northern Iran. Munis entomology and zoology, 3(1), 275-284. strongly support the factor of establishment of syrphids as alternative pollinator from higher elevational landscape of Himachal Pradesh.

\section{Acknowledgement}

We wish to express our thanks to Dr. Kailash Chandra, Director, Zoological Survey of India and Ministry of Environment, Forests and Climate Change, for funding the project and providing the necessary facilities. Thanks are due to Dr. C. Raghunathan, Div. In Charge, Ent. Div. B, for his continuous encouragement. Further, we acknowledge and convey our sincere thanks to Sri. P. Parui, Retd. Assistant Zoologist, for helping with the identification, last but not the least we express our gratitude to our fellow team members of Diptera Section, for their constant encouragement.

\section{Conflicts of Interest}

The authors declare that there are no conflicts of interest regarding the publication of this work.

Ghorpadé, K., \& Shehzad, A. (2013). An annotated checklist and select bibliography of the Hoverflies (Diptera-Syrphidae) of Pakistan, Indian subcontinent. Colemania, 37, 1-26.

Joshi, R., Sharma, P., Sharma, V., Prasad, R., Sud, R. K., \& Gulati, A. (2013). Analysis of the essential oil of large cardamom (Amomum subulatum Roxb.) growing in different agro-climatic zones of Himachal Pradesh, India. Journal of the Science of Food and Agriculture, 93(6), 1303-1309.

Mani, M. S. (2013). Ecology and biogeography of high altitude insects (Vol. 4). Springer Science \& Business Media.

Maity A., Naskar A., Hazra S., Sengupta J., Parui P., Homechaudhuri S. \& Banerjee D. 2016. New distributional records of Tabanidae (Insecta: Diptera) from different geo-climatic regions of West Bengal, India. J. Entomol. Zool. Stud., 4:1291-1298.

Miranda, G. F. G., Young, A. D., Locke, M. M., Marshall, S. A., Skevington, J. H., \& Thompson, F. C. (2013). Key to the genera of Nearctic Syrphidae. Canadian Journal of Arthropod Identification, 23(1), 351.https://doi.org/10.3752/cjai.2013.23

Naskar A., Maity A., Homechaudhuri S. \& Banerjee D. 2019. New Distributional Records of Robber Flies (Insecta: Diptera: Asilidae) From The Darjeeling 
Himalaya of West Bengal. Mun. Ent. Zool, 14(2):412426.

Potts, S. G., Petanidou, T., Roberts, S., O'Toole, C., Hulbert, A., \&Willmer, P. (2006). Plant-pollinator biodiversity and pollination services in a complex Mediterranean landscape. Biological conservation, 129(4), 519 529.https://doi.org/10.1016/j.biocon.2005.11.019

Sengupta, J., Naskar, A., Maity, A., Hazra, S., Mukhopadhyay, E., Banerjee, D., \& Ghosh, S. (2016a). An updated distributional account of Indian hover flies (Insecta: Diptera: Syrphidae). J EntomolZool Stud, 4, 381-396.

Sengupta, J., Naskar, A., Maity, A., Hazra, S., \& Banerjee, D. (2016b). New Distributional Records and Annotated Keys of Hover Flies (Insecta: Diptera: Syrphidae) from Himachal Pradesh, India. J. Adv. Zool, 37(1), 31-54.

Sengupta, J., A. Naskar, A. Maity, S. chaudhuri, and D. Banerjee. "A Taxonomic Account of Hover Flies (Insecta: Diptera: Syrphidae) With $4 \mathrm{New}$ Records from Cold Dry Zones of Himachal Pradesh, India". International Journal of Advancement in Life Sciences Research, 1(4), 13-30. https://doi.org/10.31632/ijalsr.2018v01i04.003
Int J Adv Life Sci Res. Volume 2(4)21-37 Sengupta J, Naskar A and Banerjee D, 2019.Pollinating Diptera: The Forgotten Superhero. ENVIS Newsletter. 25 (1-4): 34-36.

Ssymank, A., Kearns, C. A., Pape, T., \& Thompson, F. C. (2008). Pollinating flies (Diptera): a major contribution to plant diversity and agricultural production. Biodiversity, 9(1-2), 86-89. https://doi.org/10.1080/14888386.2008.9712892

Teskey, H.J., Vockeroth, J.R. \& Wood, D.M., (1981). Manual of Nearctic Diptera. Ottawa, Research Branch, Agriculture Canada, Monograph, 27.

Thompson, F. C., Vockeroth, J. R., \& Speight, M. C. (1982). The Linnaean species of flower flies (Diptera: Syrphidae).

Vockeroth, J. R. (1992). The flower flies of the subfamily Syrphinae of Canada, Alaska, and Greenland: Diptera, Syrphidae (Vol. 1867). Agriculture Canada.

http://publications.gc.ca/pub?id=9.811395\&sl=0

Vockeroth, J. R., Thompson, F. C., McAlpine, J. F., Peterson, B. V., Shewell, G. E., Teskey, H. J., \& Wood, D. M. (1987). Syrphidae, pp. 713-743. Manual of Nearctic Diptera, 2.

http://www.publications.gc.ca/pub?id=9.610315\&sl=0 